\title{
BMJ
}

\section{Longitudinal community plasma HIV-1 RNA concentrations and incidence of HIV-1 among injecting drug users: prospective cohort study}

\author{
Evan Wood, research scientist, ${ }^{1}$ Thomas Kerr, research scientist, ${ }^{1}$ Brandon D L Marshall, PhD candidate, ${ }^{2}$ \\ Kathy Li, senior statistician, ${ }^{1}$ Ruth Zhang, statistician, ${ }^{1}$ Robert S Hogg, director, HIV/AIDS drug treatment \\ program, ${ }^{1}$ P Richard Harrigan, director, research laboratories, Julio S G Montaner, head ${ }^{3}$
}

British Columbia Centre for

Excellence in HIV/AIDS, Vancouver, Canada

${ }^{2}$ School of Population and Public Health, University of British

Columbia, Vancouver

${ }^{3}$ Division of AIDS, Department of Medicine, University of British

Columbia, Vancouver

Correspondence to: E Wood

uhri-ew@cfenet.ubc.ca

Cite this as: $B M J$ 2009;338:b1649 doi:10.1136/bmj.b1649

\section{ABSTRACT}

Objective To examine the relation between plasma HIV-1 RNA concentrations in the community and HIV incidence among injecting drug users.

Design Prospective cohort study.

Setting Inner city community in Vancouver, Canada.

Participants Injecting drug users, with and without HIV, followed up every six months between 1 May 1996 and 30 June 2007.

Main outcome measures Estimated community plasma HIV-1 RNA in the six months before each HIV negative participant's follow-up visit. Associated HIV incidence.

Results Among 622 injecting drug users with HIV, 12435 measurements of plasma HIV-1 RNA were obtained. Among 1429 injecting drug users without HIV, there were 155 HIV seroconversions, resulting in an incidence density of 2.49 ( $95 \%$ confidence interval 2.09 to 2.88 ) per 100 person years. In a Cox model that adjusted for unsafe sexual behaviours and sharing used syringes, the estimated community plasma HIV-1 RNA concentration remained independently associated with the time to HIV seroconversion (hazard ratio 3.32 (1.82 to 6.08, P<0.001), per $\log _{10}$ increase). When the follow-up period was limited to observations after 1 January 1988 (when the median plasma HIV RNA concentration was $<20000$ copies $/ \mathrm{ml}$ ), the median viral load was no longer statistically associated with HIV incidence (1.70 (0.79 to 3.67, $P=0.175$ ), per $\log _{10}$ increase).

Conclusions A longitudinal measure of community plasma HIV-1 RNA concentration was correlated with the community HIV incidence rate and predicted HIV incidence independent of unsafe sexual behaviours and sharing used syringes. If these findings are confirmed, they could help to inform both HIV prevention and treatment interventions.

\section{INTRODUCTION}

Since the mid-1990s, there have been major advances in the medical management of HIV infection. In particular, highly active antiretroviral therapy (HAART) has led to substantial reductions in HIV related morbidity and mortality. ${ }^{1}$ As HAART is known to effectively reduce a patient's plasma HIV RNA concentration, ${ }^{2}$ it has been debated whether or not antiretroviral therapy can reduce HIV transmission. ${ }^{3-6}$ While several mathematical models have suggested this might be the case, researchers have suggested that the increasing use of HAART might lead to increased HIV risk behaviour, which could overwhelm the possible protective effect of HAART. ${ }^{3-6}$ Empirical data are urgently needed to inform this controversy, particularly given the recent negative results of clinical trials involving leading candidates in the microbicide and vaccine specialties. ${ }^{78}$ The topic has been a focus of recent academic meetings of HIV experts, ${ }^{9}$ and the World Health Organization recently released a consensus statement in which using HAART for HIV prevention was identified as a top policy-relevant research question. ${ }^{10}$

To date, no study has described the real world relation between community plasma HIV RNA concentrations and HIV incidence. This is an important gap in knowledge because the potential of HAART to reduce HIV incidence has attracted growing international interest, ${ }^{5611}$ and the ongoing debates over the possible impact of increasing access to HAART might be hindering global efforts to increase its use. $^{612}$ We therefore tested the hypothesis that a longitudinal estimate of community plasma HIV-1 RNA concentration would be associated with an estimate of community HIV incidence independent of HIV risk behaviours, such as sharing used syringes and engaging in unsafe sexual practices.

\section{DESIGN}

Between 1 May 1996 and 30 June 2007, injecting drug users with and without HIV were recruited into a prospective cohort study from the Downtown Eastside neighbourhood of Vancouver, Canada. Individuals were recruited regardless of HIV status and followed up every six months. Two virtual cohorts were created at the database level to independently assess incidence of HIV and plasma viral load. Those without HIV at baseline made up the Vancouver Injection Drug Users 
Study (VIDUS), an open prospective cohort of injecting drug users described in detail previously. ${ }^{1314}$ Those with HIV-1 made up another cohort known as the Barriers to Accessing Antiretroviral Therapy (BART). ${ }^{1516}$ Briefly, at baseline participants gave blood samples for HIV serology and completed a questionnaire administered by an interviewer and then returned every six months for follow-up evaluation. Extensive outreach methods with snowball sampling techniques ${ }^{17} 18$ were used to derive a representative sample. Individuals who agreed to participate were offered $\$ \mathrm{C} 20$ at each study visit. Because of the extent of poverty in the neighbourhood, as well as the nursing and support services offered through the study, the estimated refusal rate for participation in the study was under $10 \% .{ }^{19}$

\section{Community plasma HIV-1 RNA concentrations}

We estimated community plasma HIV-1 RNA concentrations every six months using data from BART cohort participants recruited from 1 May 1996 to 30 June 2007. Plasma HIV-1 RNA concentration was measured with the Roche Amplicor Monitor assay (Roche Molecular Systems, Mississauga, Canada). All plasma HIV-1 RNA samples collected from BART participants at follow-up were used to derive a longitudinal estimate of the plasma HIV-1 RNA concentration in the community over time. As previously described, ${ }^{1516}$ the local setting is quite unusual in that there is a centralised antiretroviral dispensation programme and HIV/AIDS laboratory, allowing for a complete prospective profile of plasma HIV-1 RNA levels and use of antiretroviral therapy among cohort participants.

In addition, to determine explanations for changing concentrations of community plasma HIV-1 RNA during follow-up, we assessed use of antiretrovirals among BART participants during each year of the study and analysed changes during the study period using the Mantel test for trend.

\section{Community HIV-1 incidence}

We used similar standardised follow-up methods every six months (that is, twice a year) to estimate the annual

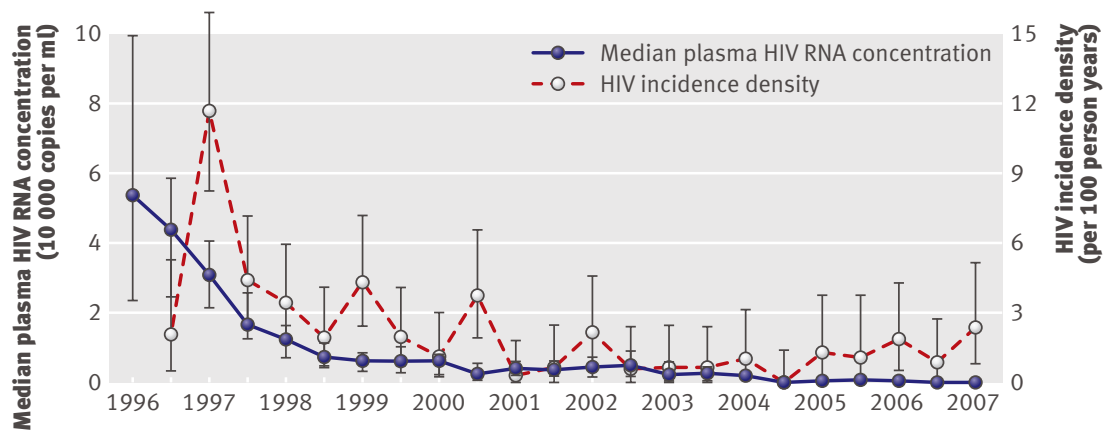

Year

Estimated community plasma HIV-1 RNA concentrations and HIV incidence density, with $95 \%$ confidence intervals, among two parallel cohorts of injecting drug users. HIV incidence first estimated in second half of 1996 as enrolment started in May 1996 and repeat HIV tests to assess incidence were available only after six months of follow-up
HIV incidence among participants recruited into the VIDUS cohort. As previously described, ${ }^{14} \mathrm{HIV}$ infection was assessed at each follow-up visit, and the date of HIV seroconversion was estimated with the midpoint between the last negative and the first positive antibody test result. We considered all participants with at least one follow-up visit to determine HIV incidence, and participants who remained persistently HIV seronegative were censored at the time of their most recent HIV antibody test result before 30 June 2007. HIV tests reactive on enzyme linked immunosorbent assay at St Paul's Hospital were confirmed by western blotting at the British Columbia Centre for Disease Control.

\section{Statistical analyses}

To assess for a possible crude correlation between the twice a year estimates of community plasma HIV-1 RNA and the twice a year estimates of community HIV incidence, we first divided the study period into six month intervals and used the Spearman rank order correlation coefficient to assess for statistical correlation. In addition, to present the data visually, we also plotted the median plasma HIV RNA concentrations and the incidence density every six months for each year of the study and presented the uncertainty around these estimates by calculating 95\% confidence inter vals. ${ }^{20}$ For the estimates of HIV incidence, we calculated 95\% confidence intervals using the Poisson distribution. For the estimates of median plasma HIV-RNA concentration, we calculated non-parametric two sided $95 \%$ confidence intervals by non-parametric methods based on the ordered plasma HIV-1 RNA concentrations.

We also used Cox proportional hazards regression to assess factors associated with the time to HIV infection and examined whether the estimated community plasma HIV-1 RNA concentration in the six months before each participant's follow-up visit was associated with HIV-1 incidence, while adjusting for HIV risk behaviour. Specifically, to assess for potential confounding, we calculated unadjusted and adjusted hazard ratios of HIV infection per $\log _{10}$ increase in the estimated community plasma HIV-1 RNA concentration. The multivariate model was fitted with an a priori defined modelling approach whereby we examined the hazard ratio for HIV-1 RNA after adjustment for HIV risk behaviours and previously identified potential confounders. Specifically, we adjusted for sharing used syringes (yes versus no), unsafe sex (insertive or receptive anal or vaginal intercourse without a condom versus no unsafe sex), ethnicity (white versus other), cocaine use ( $\geq$ daily versus $<$ daily), heroin use ( $\geq$ daily versus $<$ daily), and unstable housing (living in a single room occupancy hotel, shelter, recovery or transition house, jail, on the street, or having no fixed address versus having stable housing). As with the estimated community plasma HIV-1 RNA concentration, all behavioural variables were time updated based on the data of the twice a year questionnaire and refer to the six months before the interview. All behavioural 
Table 1|Baseline demographic characteristics of injecting drug users with and without HIV. Figures are numbers (percentages) of participants unless stated otherwise

\begin{tabular}{lcc} 
Characteristic & $\begin{array}{c}\text { HIV positive* } \\
(\mathrm{n}=622)\end{array}$ & $\begin{array}{c}\text { HIV negative† } \\
(\mathrm{n}=1429)\end{array}$ \\
$\begin{array}{l}\text { Median }(\mathrm{IQR}) \text { age } \\
\text { (years) }\end{array}$ & $36.6(30.3-43.3)$ & $36.1(27.2-42.4)$ \\
\hline Male & $372(59.8)$ & $965(67.5)$ \\
\hline Female & $250(40.2)$ & $464(32.5)$ \\
\hline Ethnicity: & $352(56.6)$ & $899(62.9)$ \\
\hline White & $270(43.4)$ & $530(37.1)$ \\
\hline Other & & \\
\hline
\end{tabular}

$\mathrm{IQR}=$ interquartile range.

*Cohort used to estimate longitudinal pattern of plasma viral load. †Cohort used to evaluate HIV incidence in community.

variable definitions were identical to previous reports. ${ }^{1314}$ To assess for explanations for changes in plasma HIV RNA concentrations over time, we linked participants to the local province-wide antiretroviral dispensation programme to examine patterns of antiretroviral use during the study period. Analyses were conducted with SAS 9.1 (Cary, NC) and the threshold for significance was set at $\mathrm{P}<0.05$. All $\mathrm{P}$ values were two sided.

\section{RESULTS}

Table 1 shows the baseline characteristics of the cohorts of participants with and without HIV. The baseline age was similar between the two cohorts (36.6 v 36.1 years), whereas those with HIV were more likely to be female $(250(40.2 \%) v 464(32.5 \%))$ and of non-white ethnicity $(270(43.4 \%) v 530(37.1 \%))$.

\section{Community plasma HIV-1 RNA}

During the study period, 622 BART participants underwent plasma HIV-1 RNA assessments, among whom $250(40.2 \%)$ were women and the median age was 37 (interquartile range 30-43). Among the 622 participants, there were 12435 plasma HIV-1 RNA assessments, with a median of 17 (8-31) measurements per person. The figure shows the estimated median plasma HIV-1 RNA concentrations every six months for 1996-2007.

Table 2 |Cox proportional hazards regression of time to HIV infection among 1429 HIV negative injecting drug users followed from 1 May 1996 to 30 June 2007

\begin{tabular}{|c|c|c|}
\hline Characteristic & Relative hazard $(95 \% \mathrm{Cl})$ & $P$ value \\
\hline Plasma HIV RNA (per $\log _{10}$ increase)* & 3.32 (1.82 to 6.08$)$ & $<0.001$ \\
\hline Unsafe sex† (yes $v$ no) $\ddagger$ & $1.09(0.77$ to 1.54$)$ & 0.619 \\
\hline Used syringe sharing (yes v no) & $1.45(0.99$ to 2.12$)$ & 0.058 \\
\hline Ethnicity (white $v$ other) & $0.65(0.47$ to 0.91$)$ & 0.011 \\
\hline Heroin injection ( $\geq$ daily $v<$ daily) $\ddagger$ & $1.35(0.97$ to 1.90$)$ & 0.079 \\
\hline Cocaine injection ( $\geq$ daily $v<$ daily) $\ddagger$ & $2.50(1.76$ to 3.54$)$ & $<0.001$ \\
\hline Unstable housing (yes $v$ no)§ & 1.41 (1.00 to 1.98$)$ & 0.049 \\
\hline
\end{tabular}

*Plasma HIV RNA was time updated based on median value in BART cohort during six months before each HIV negative participant's follow-up visits.

†Defined as insertive or receptive vaginal or anal intercourse.

$\ddagger$ All behavioural data time updated based on data from follow-up every six months.

§Living in single room occupancy hotel, shelter, recovery or transition house, jail, on street, or having no fixed address.

\section{HIV-1 incidence}

During the study period, 1796 individuals who were HIV negative at baseline were enrolled into the VIDUS cohort. Of these, 367 (20.4\%) were lost to follow-up before re-assessment of HIV infection and were therefore excluded from further analysis. Those lost to follow-up were more likely to be white $(\mathrm{P}=0.012)$, but there were no age or sex differences (both $\mathrm{P}>0.05$ ). Among the 1429 individuals included in the HIV incidence analyses, $464(32.5 \%)$ were women, the median age was 36.1 (27.2-42.4), and the median number of follow-up visits was 8 (3-16). During the study period, there were $155 \mathrm{HIV}$ seroconversions, resulting in an overall incidence density of 2.49 (95\% confidence interval 2.09 to 2.88 ) per 100 person years. The figure shows the incidence density for every six months for 1996-2007.

\section{HIV-1 RNA and HIV incidence}

As implied by the patterns in the figure, when we divided the 11 year study period into 22 six month intervals, the median plasma HIV-1 RNA concentration and the HIV-1 incidence were correlated (Spearman correlation coefficient $0.48 ; \mathrm{P}=0.024$ ).

In unadjusted Cox regression analyses, we found that the median estimated community plasma HIV-1 RNA concentration during the six months before each HIV negative participant's follow-up visit was associated with HIV seroconversion (hazard ratio 3.57 (2.03 to 6.27), $\mathrm{P}<0.001$, per $\log _{10}$ increase). As shown in table 2 , in a multivariate model that adjusted for sharing used syringes, unsafe sex, ethnicity, daily cocaine use, daily heroin use, and unstable housing, the median plasma HIV-1 RNA concentration during the six months before each HIV negative participant's follow-up visit remained independently associated with the time to HIV seroconversion (hazard ratio 3.32 (1.82 to 6.08$), \mathrm{P}<0.001$, per $\log _{10}$ increase). In a post-hoc analysis, which limited follow-up to the period after 1 January 1988 (when the median plasma HIV RNA concentration was $<20000$ copies/ml), we found that the median viral load was no longer significantly associated with HIV incidence $(1.70$ (0.79 to 3.67), $\mathrm{P}=0.175$, per $\log _{10}$ increase).

When we assessed for explanations for the observed longitudinal pattern of estimated community plasma HIV-1 RNA (figure), we found that the number of individuals who had started antiretroviral therapy increased during the study period from $42.5 \%$ in 1996 to $69.6 \%$ in 2007 (Mantel test for trend across all 11 years $\mathrm{P}<0.001)$. Among those receiving antiretroviral drugs, we found that the number using at least three antiretroviral drugs (versus fewer than three drugs) in their antiretroviral regimen increased during the study period from $8.4 \%$ in 1996 to $98.8 \%$ in 2007 (Mantel test for trend across all 11 years $\mathrm{P}<0.001)$.

\section{DISCUSSION}

In a small urban neighbourhood with high rates of injecting drug use, we found that estimated community 
plasma HIV-1 RNA concentration predicted estimated community HIV incidence, and that this association was independent of HIV risk behaviours and other potential confounders. Previous studies have suggested that injecting drug users might be less likely to access antiretroviral therapy because of physicians' concerns related to lifestyle issues and reduced antiretroviral adherence. ${ }^{21}$ If our findings are confirmed, outreach strategies could be used to improve access to HAART among this population in an effort to reduce the HIV incidence. Any benefit of earlier use of HAART, however, must be balanced with antiretroviral toxicities and potential for increased antiretroviral resistance. ${ }^{22}$

\section{Comparison with other studies}

Our study was observational, and the observed declines in HIV incidence in the community might not be causally related to the observed decline in the estimated community plasma viral load. It is nonetheless compelling that the effect of plasma HIV-1 RNA on HIV incidence was independent of time updated measurements of HIV risk behaviours, drug use patterns, and other possible confounders. Several lines of evidence support the hypothesis that community plasma HIV-1 RNA concentrations might be among the factors that determine community HIV incidence. Most importantly, HAART has been shown to reduce HIV-1 RNA concentrations in blood, ${ }^{23}$ the female genital tract, ${ }^{24}$ the rectum, ${ }^{25}$ and semen, ${ }^{26}$ which might make those with HIV less likely to transmit the virus. ${ }^{5}$ An ecological study from Taiwan showed that the expanded use of HAART was associated with a greater than $50 \%$ reduction in new HIV cases. ${ }^{27}$ Similarly, a study from Uganda, which examined couples serodiscordant for HIV, found no cases of HIV transmission where the index case had an HIV-1 RNA concentration below 1500 copies $/ \mathrm{ml}^{28}$ Interestingly, we found that the association between plasma viral load and HIV incidence was no longer significant in subanalyses restricted to the period when the median viral load reached $<20000$ copies/ml. This finding introduces some uncertainty into our overall finding as it suggests that our results are largely driven by the early years, during which the plasma viral load was high. The relation between viral load and HIV incidence might be less strong when viral load is below a certain threshold. Alternatively, as this is an observational study, an unmeasured confounding variable might explain the association, and the results in this subanalysis might not have been consistent because this confounding variable did not have a consistent effect throughout the follow-up period.

\section{Strengths and limitations}

Our analyses were also limited by the fact that there is a known delay between HIV exposure and seroconversions, and, consistent with most studies on HIV incidence, we had to estimate an individual's date of HIV seroconversion as the midpoint between the last negative HIV test and the first positive test. ${ }^{14}$ As a result,
HIV seroconversions might have happened slightly earlier than the year to which they were assigned in our study. Regarding this limitation, it is interesting that the highest rate of HIV seroconversion was observed in the year after the highest community plasma HIV-1 RNA concentration. Antiretroviral resistance is unlikely to explain our findings as increased resistance is associated with use of less potent antiretrovirals and increasing viral load, ${ }^{2930}$ whereas during the study period we observed the use of more potent antiretroviral therapy and decreased plasma HIV RNA concentrations. Finally, although we used extensive outreach methods and snowball sampling techniques in an effort to derive a representative sample, ${ }^{17} 18$ injecting drug users are a highly marginalised and hidden population, and we do not know with certainty that our cohort is representative of injecting drug users in the community. Previous studies have suggested the demographics of our cohort are consistent with other samples of the city's injecting drug users, ${ }^{31}$ and it is noteworthy that our study encompasses about $30 \%$ of the estimated 4700 injecting drug users living in the neighbourhood (unpublished report, Health Canada, 1998). Although the question of the impact of a community's plasma HIV-1 RNA concentration on HIV incidence might be better answered by a study design that randomised communities to differing levels of HAART use, such an approach might present ethical challenges because of HAART's known effects on reducing mortality, and these data are not presently available.

\section{Conclusions and policy implications}

We have shown that a longitudinal measure of community plasma HIV-1 RNA concentration correlates with the community HIV incidence rate and can predict HIV incidence independent of unsafe sexual behaviours and sharing syringes. These data should prompt a re-examination of arguments that dichotomise HIV prevention and HIV treatment, as they might not be independent strategies to reduce the rate of new HIV infections. ${ }^{6}{ }^{12}$ These data should also help to inform the debates regarding global increase in use of antiretrovirals and possible unintended negative impacts of expanded HAART use on HIV risk behaviour and new HIV infections, as expanded HAART use in the community was associated with both reduced community plasma HIV RNA concentrations and subsequent HIV incidence. ${ }^{3-6}$

We thank the VIDUS and BART participants, and Deborah Graham, Tricia Collingham, and Kelly Hsu for their research and administrative assistance.

Contributors: EW designed the study, wrote the initial draft of the manuscript, and is guarantor. RZ and $\mathrm{KL}$ conducted the statistical analyses. All authors contributed to the design of the study and the drafting of the manuscript.

Funding: The VIDUS and BART studies are supported by US National Institutes of Health grants R01DA011591 and R01DA021525 and by Canadian Institutes of Health Research grant MOP-79297. TK is supported by a Canadian Institutes of Health Research (CIHR) new investigator award and a Michael Smith Foundation for Health Research (MSFHR) scholar award. BDLM is supported by a doctoral research award from CIHR and a MSFHR senior trainee award. JSGM has received an 


\section{WHAT IS ALREADY KNOWN ON THIS TOPIC}

Antiretroviral therapy reduces plasma viral load and reduces HIV related mortality

Its potential role in reducing HIV transmission by reducing an individual's infectivity is controversial

Drug misusers with HIV are less likely to access HAART (highly active antiretroviral therapy) than other people with HIV

\section{WHAT THIS STUDY ADDS}

A longitudinal measure of community plasma HIV-1 RNA correlates with the community HIV incidence rate and predicts HIV incidence independent of unsafe sexual behaviours and sharing syringes

These data should prompt a re-examination of arguments that dichotomise HIV prevention and HIV treatment, which might not be independent strategies for reducing the rate of new HIV infections

Injecting drug users can be successfully attracted to and retained in HAART programmes

Avant-Garde award (DP1 DA026182) from the National Institute of Drus Abuse, National Institutes of Health. The authors affirm the independence of the researchers from the funders.

Competing interests: RSH has received funding for research and continuing medical education programmes from pharmaceutical companies, including Abbott, Boehringer Ingelheim, and GlaxoSmithKline. JSGM has received educational grants from, served as an ad hoc adviser to, or spoken at various events sponsored by Abbott Laboratories, Agouron Pharmaceuticals, Boehringer Ingelheim Pharmaceuticals, Borean Pharma AS, Bristol-Myers Squibb, DuPont Pharma, Gilead Sciences, GlaxoSmithKline, Hoffmann-La Roche, Immune Response Corporation, Incyte, Janssen-Ortho, Kucera Pharmaceutical Company, Merck Frosst Laboratories, Pfizer Canada, Sanofi Pasteur, Shire Biochem, Tibotec Pharmaceuticals, and Trimeris.

Ethical approval: The research was approved by the University of British Columbia's research ethics board at St Paul's Hospital.

1 Palella FJ Jr, Delaney KM, Moorman AC, Loveless MO, Fuhrer J, Satten GA, et al. Declining morbidity and mortality among patients with advanced human immunodeficiency virus infection. HIV Outpatient Study Investigators. N Engl J Med 1998;338:853-60.

2 Hammer SM, Squires KE, Hughes MD, Grimes JM, Demeter LM, Currier JS, et al. A controlled trial of two nucleoside analogues plus indinavir in persons with human immunodeficiency virus infection and CD4 cell counts of 200 per cubic millimeter or less. AIDS Clinical Trials Group 320 Study Team. N Engl J Med 1997;337:725-33.

3 Blower SM, Gershengorn HB, Grant RM. A tale of two futures: HIV and antiretroviral therapy in San Francisco. Science 2000;287:650-4.

4 Velasco-Hernandez JX, Gershengorn HB, Blower SM. Could widespread use of combination antiretroviral therapy eradicate HIV epidemics? Lancet Infect Dis 2002;2:487-93.

5 Cohen MS, Gay C, Kashuba AD, Blower S, Paxton L. Narrative review: antiretroviral therapy to prevent the sexual transmission of HIV-1. Ann Intern Med 2007;146:591-601.

6 Montaner JS, Hogg R, Wood E, Kerr T, Tyndall M, Levy AR, et al. The case for expanding access to highly active antiretroviral therapy to curb the growth of the HIV epidemic. Lancet 2006;368:531-6.

7 HIV vaccine failure prompts Merck to halt trial. Nature 2007;449:390.

8 Cohen J. AIDS research. Microbicide fails to protect against HIV. Science 2008:319:1026-7.

9 Steinbrook R. The AIDS epidemic-a progress report from Mexico City. N Engl / Med 2008;359:885-7.
10 World Health Organization. Consensus statement: addressing knowledge gaps in the public health approach to delivering antiretroviral therapy and care. www.iasociety.org/Web/ WebContent/File/Consensus\%20Statement\%2030-07-08.pdf.

11 Aceijas C, Oppenheimer E, Stimson GV, Ashcroft RE, Matic S, Hickman M. Antiretroviral treatment for injecting drug users in developing and transitional countries 1 year before the end of the "Treating 3 million by 2005. Making it happen. The WHO strategy" ("3 by 5"). Addiction 2006;101:1246-53.

12 Marseille E, Hofmann PB, Kahn JG. HIV prevention before HAART in sub-Saharan Africa. Lancet 2002;359:1851-6.

13 Kerr T, Tyndall M, Li K, Montaner JS, Wood E. Safer injection facility use and syringe sharing in injection drug users. Lancet 2005;366:316-8

14 Wood E, Lloyd-Smith E, Li K, Strathdee SA, Small W, Tyndall MW, et al. Frequent needle exchange use and HIV incidence in Vancouver, Canada. Am / Med 2007;120:172-9.

15 Strathdee SA, Palepu A, Cornelisse PG, Yip B, O’Shaughnessy MV, Montaner JS, et al. Barriers to use of free antiretroviral therapy in injection drug users. JAMA 1998;280:547-9.

16 Wood E, Hogg RS, Bonner S, Kerr T, Li K, Palepu A, et al. Staging for antiretroviral therapy among HIV-infected drug users. JAMA 2004;292:1175-7.

17 Dunn J, Ferri CP. Epidemiological methods for research with drug misusers: review of methods for studying prevalence and morbidity Rev Saude Publica 1999;33:206-15.

18 Lopes CS, Rodrigues LC, Sichieri R. The lack of selection bias in a snowball sampled case-control study on drug abuse. Int J Epidemiol 1996;25:1267-70.

19 Strathdee SA, Patrick DM, Currie SL, Cornelisse PG, Rekart ML, Montaner JS, et al. Needle exchange is not enough: lessons from the Vancouver injecting drug use study. AIDS 1997;11:F59-65.

20 Collett, D. Modelling survival data in medical research. New York: Chapman \& Hall, 1994.

21 Loughlin A, Metsch L, Gardner L, Anderson-Mahoney P, Barrigan M, Strathdee S. Provider barriers to prescribing HAART to medicallyeligible HIV-infected drug users. AIDS Care 2004;16:485-500.

22 Wood E, Hogg RS, Harrigan PR, Montaner JS. When to initiate antiretroviral therapy in HIV-1-infected adults: a review for clinicians and patients. Lancet Infect Dis 2005;5:407-14.

23 Wood E, Hogg RS, Yip B, Moore D, Harrigan PR, Montaner JS. Impac of baseline viral load and adherence on survival of HIV-infected adults with baseline CD4 cell counts > or $=200$ cells $/ \mathrm{microl}$. AIDS 2006;20:1117-23.

24 Cu-Uvin S, Caliendo AM, Reinert S, Chang A, Juliano-Remollino C, Flanigan TP, et al. Effect of highly active antiretroviral therapy on cervicovaginal HIV-1 RNA. AIDS 2000;14:415-21.

25 Lampinen TM, Critchlow CW, Kuypers JM, Hurt CS, Nelson PI, Hawes SE, et al. Association of antiretroviral therapy with detection of HIV-1 RNA and DNA in the anorectal mucosa of homosexual men. AIDS 2000;14:F69-75.

26 Vernazza PL, Gilliam BL, Flepp M, Dyer JR, Frank AC, Fiscus SA, et al. Effect of antiviral treatment on the shedding of HIV-1 in semen. AIDS 1997;11:1249-54.

27 Fang CT, Hsu HM, Twu SJ, Chen MY, Chang YY, Hwang JS, et al. Decreased HIV transmission after a policy of providing free access to highly active antiretroviral therapy in Taiwan. J Infect Dis 2004;190:879-85

28 Quinn TC, Wawer MJ, Sewankambo N, Serwadda D, Li C, Wabwire-Mangen F, et al. Viral load and heterosexual transmission of human immunodeficiency virus type 1. Rakai Project Study Group. N Engl J Med 2000;342:921-9.

29 Martinez-Picado J, DePasquale MP, Kartsonis N, Hanna GJ, Wong J, Finzi D, et al. Antiretroviral resistance during successful therapy of HIV type 1 infection. Proc Natl Acad Sci U S A 2000;97:10948-53.

30 Gallego 0, de Mendoza C, Perez-Elias MJ, Guardiola JM, Pedreira I, Dalmau D, et al. Drug resistance in patients experiencing early virological failure under a triple combination including indinavir. AIDS 2001;15(13):1701-6.

31 Tyndall MW, Craib KJ, Currie S, Li K, O’Shaughnessy MV, Schechter MT. Impact of HIV infection on mortality in a cohort of injection drug users. J Acquir Immune Defic Syndr 2001;28:351-7.

Accepted: 12 January 2009 